\title{
Una aproximación al turismo Slow \\ El turismo Slow en las Cittaslow de España
}

\author{
Andrea de Luis Blanco \\ andreadeluis@gmail.com
}

\section{RESUMEN}

En un presente en el que el tiempo lícito de ocio aumenta progresivamente, y que cada vez más está condicionado por la preocupación ambiental, surgen nuevas formas de entender el turismo. El turismo Slow nace de la filosofía Slow, en el seno de la teoría del decrecimiento, como alternativa a la actual práctica turística.

El presente trabajo tiene como objetivo arrojar algo de luz a este tema a través del análisis de las principales particularidades que caracterizan esta filosofía turística. Asimismo, se ha pretendido complementar este objetivo con un pequeño estudio empírico. Para ello, a través de entrevistas en profundidad hemos querido comprobar si las localidades integradas en Cittaslow, Red Estatal de los municipios por la calidad de vida, enmarcada también en la filosofía del movimiento Slow, son adecuadas para la práctica del objeto de estudio de este trabajo, el turismo Slow.

Una vez obtenidos los resultados, y tras su análisis, se ha llegado a la conclusión de que los municipios adheridos a esta red no muestran una realidad turística focalizada en los principios del turismo Slow, sino que, debido a su corta historia dentro de la red Cittaslow y a la tradición turística que alguno de ellos tienen, muestran unas características de oferta y demanda bastante alejadas de la filosofía Slow.

Palabras clave: Decrecimiento; ocio; movimiento Slow. 


\title{
An approach for Slow tourism \\ Slow tourism in Spain's Cittaslow
}

\author{
Andrea de Luis Blanco \\ andreadeluis@gmail.com
}

\section{ABSTRACT}

At the present time, when leisure time is increasing and it is influenced by the concern of environmental issue, new ways of understanding tourism are emerging. Slow tourism arises from Slow philosophy, within the economic degrowth theory, as an alternative to the current tourist activity.

The aim of this document is to shed some light on this subject through the analysis of the main special features that characterize this tourist philosophy. We also wanted to complement this objective with a little empirical study. Through in-depth interviews to people from Spanish Cittaslow, State Network of cities for quality of life, which it is also into the Slow movement philosophy, we tried to know if these cities are suitable for doing Slow tourism.

After obtaining the results and analyzing them we came to the conclusion that tourism in the Spanish cities included in the network is not focused on a Slow tourism basis, indeed some of them have supply and demand characteristics which are quite far from the Slow philosophy due to their short history as members of the Cittaslow network and their long tourist activity tradition.

Keywords: Degrowth; leisure; Slow movement. 


\section{INTRODUCCIÓN}

\section{El ocio turístico desde una perspectiva social}

La sociedad actual se ha convertido en una idólatra del tiempo. El tiempo viene a convertirse en el eje central de las actividades del ser humano desde la Revolución Industrial. Su programación, especialmente a manos de los empresarios, dio paso a un nuevo estilo de vida caracterizado por la sumisión a las exigencias del reloj. Desde entonces, el tiempo se ha convertido en un factor competitivo que gobierna tanto la vida laboral del hombre como su vida privada. Queremos hacer más en menos tiempo; creemos que lo rápido es lo más adecuado e inconscientemente aumentamos la velocidad de nuestros actos. Sin embargo, a pesar de todos los avances tecnológicos que nos ofrecen tiempo extra y de los avances en medicina que nos permiten vivir un mayor número de años, cada vez más aceleramos el ritmo de vida y, sin embargo, tenemos la sensación de disponer de menos tiempo (Honoré, 2009).

Se puede decir que nos encontramos inmersos en la cultura de la velocidad y del ahorro del tiempo, de la competitividad, del fomento del consumismo, de la valoración material de las cosas, etc. Surge así un sistema social cuyos valores predominantes se basan en la economía. Las consecuencias de asumir tal velocidad repercuten tanto en la vida laboral como en la vida privada y se traducen en problemas de improductividad, consumismo y especialmente en problemas de salud, tanto física como mental.

De esta forma, cabe esperar que el tiempo libre y de ocio, especialmente bajo la fórmula de turismo, sean propicios para que el hombre se recupere física y mentalmente, donde se desarrolle como ser humano y donde encuentre evasión lúdica de su rutina diaria. Sin embargo, a lo largo de las distintas organizaciones sociales el ocio, y por ende el turismo, han estado por mucho tiempo destinados a las élites sociales y no siempre han desarrollado la misma función social.

Tras largos años en los que muchos hombres -esclavos, agricultores, obreros, etc.vivían su tiempo libre bajo represión, se libró finalmente la batalla para conquistarlo. Fue fundamentalmente una batalla por la conquista de la libertad. Su consecución y la aparición de la civilización del ocio fueron posibles en gran parte debido a la tecnificación del proceso de producción. Posteriormente, la sociedad de consumo, al propiciar más tiempo libre a los productores, va creando nuevas necesidades de ocio, que van incrementando a su vez el consumo, motor finalmente del desarrollo económico. Podría decirse que "[l]a sociedad de consumo y la civilización del ocio serían la cara y la cruz de la misma realidad" (Zorrilla, 1990: 126).

Por su parte, paralelo a la evolución del ocio y el tiempo libre, el turismo como consumo no sólo alimenta el sistema económico sino que le confiere al hombre un status social, un símbolo de clase, reflejo de su bienestar material. No obstante, el consumo turístico también se configura como expresión de la personalidad del turista, de sus preferencias y gustos, de su estilo de vida. 
Sea como sea, el turismo es una evasión de la vida alienante hacia la libertad pero es también consumo y como tal implica alienación. La solución pasa no por la simple crítica de la sociedad actual sino que "hay que denunciar, en suma, la justificación inmanente del consumo, su conversión en fin último y supremo" (Castillo, 1987, citado por Álvarez Sousa, 1994: 337).

\section{El movimiento Slow: Contextualización e historia}

Vivimos en una sociedad de consumo, inmersa dentro de la ideología de la economía de crecimiento, esto es, el sistema de organización económica orientado, objetiva o deliberadamente, hacia la maximización del crecimiento económico (Latouche, 2008: 35). En este marco, el crecimiento económico parece haberse configurado como panacea resolutiva de los grandes problemas sociales: reducción de la pobreza, cohesión social, servicios mínimos garantizados, reducción del desempleo, etc. Pero nada más lejos de la realidad: el crecimiento se ha convertido en sinónimo de desigualdades sociales, estandarización y homogenización cultural, contaminación, pérdida de recursos naturales y asociación de la felicidad y el bienestar con el consumo.

No obstante, el mayor efecto negativo del actual sistema de crecimiento parece afectar a nivel individual, concretamente a nivel mental, en la forma de considerar la realidad. Así lo mencionábamos anteriormente cuando hablábamos de una sociedad como la nuestra, fundamentada en valores económicos; una sociedad cuyo modo de vida induce constantemente al consumismo como medio para alcanzar la felicidad, falacia a la que ningún nivel de despilfarro parece alcanzar.

Se hace necesario, por tanto, encontrar una solución, un cambio de paradigma que permita hacernos ver la necesidad de abandonar el ilógico objetivo del crecimiento por el crecimiento. Llegados a este punto, varios autores vienen gestando desde hace unos años una alternativa que le ponga freno al crecimiento ilimitado y que replantee la actual sociedad de consumo y los pilares sobre los que se basa: progreso, ciencia y técnica (Latouche, 2008).

De acuerdo con Schneider et al. (2010: 512), el decrecimiento sostenible puede ser definido como "una reducción equitativa de la producción y consumo que incremente el bienestar humano y que mejore las condiciones ecológicas a nivel local y global, a corto y largo plazo".

Los objetivos del decrecimiento pasan por "encontrar las necesidades básicas humanas y asegurar una alta calidad de vida, mientras se reduce el impacto medioambiental de la economía global a un nivel sostenible, equitativamente distribuido entre naciones ${ }^{1 "}$. La idea del decrecimiento pretende ser una transición voluntaria por lo que sólo es posible en una sociedad de decrecimiento, en una sociedad concienciada que haya abandonado el imaginario dominante del crecimiento (Latouche, 2006).

Según Norberg-Hodge (2006), para alcanzar estas metas es necesaria la desvinculación con la economía progresivamente mundializada, pero no sólo pensando a

\footnotetext{
${ }^{1}$ Véase <http://www.degrowth.eu/v1/index.php?id=56>
} 
nivel mundial y actuando localmente sino que se debe pensar y actuar simultáneamente a nivel local y global. Esto implica que paralelas a las modificaciones políticas y reglamentarias deben surgir diversas iniciativas locales.

En esta línea, son numerosas las experiencias que han surgido a lo largo de los últimos años, principalmente a nivel local pero que en algunos casos ya se han multiplicado mundialmente. Dichas prácticas intentan proporcionar respuestas a la problemática del sistema económico productivista neoliberal dentro de un contexto concreto e incluyen iniciativas de disminución del ritmo de vida, comida orgánica, estilos de vida simples, medios de transporte no contaminantes, etc.

Destacan así The Society for the Deceleration of Time [Sociedad para la Deceleración del Tiempo], el Club del Perezoso o Take your Time Back [Recupera tu tiempo]. De acuerdo con Honoré (2009), todas estas iniciativas son el reflejo de la necesidad de la sociedad actual de reducir el ritmo de vida para vivir mejor, de reemplazar el culto a la velocidad por el culto a la lentitud y así, encontrar el equilibrio necesario. La base de esta filosofía de la lentitud ha dado lugar al movimiento Slow, que si bien todavía se está formando y por tanto, no dispone de una estructura formal, es una tendencia cultural extendida mundialmente que cuestiona el materialismo propulsado por la economía globalizada. En lugar del consumismo y la velocidad en la que vivimos promueve, a través de la lentitud, una aproximación a valores cualitativos: "la filosofía del movimiento Slow proporciona las cosas que realmente nos hacen felices: buena salud, un medio ambiente en buen estado, comunidades y relaciones fuertes" (Honoré, 2009: 295).

Sea como sea, lo relevante de todas estas iniciativas del movimiento Slow es el cambio de actitud de la sociedad que puede sentar las bases sociales para el decrecimiento ya que "el decrecimiento no sólo es un proyecto científico sino parte de un movimiento social más amplio" (Schneider et al., 2010: 517).

Dentro del movimiento Slow destaca Slow Food como una de las manifestaciones más importantes que se han llevado a cabo bajo su filosofía. Fue fundado en Italia en 1986 como oposición a la Fast Food o comida rápida. Este movimiento no critica solamente un tipo de comida de baja calidad sino los procesos de producción y las pautas y hábitos de consumo que conllevan, caracterizados por la homogeneidad y estandarización que se extienden por todos los continentes alterando significativamente la cultura propia.

La idea clave es defender el derecho al placer que brindan los alimentos, lo que asimismo implica la responsabilidad de protección de la herencia cultural y de las tradiciones culinarias que hacen posible dicho placer. A partir de esa búsqueda del placer, el movimiento trabaja en una esfera más global en contra de la alta velocidad a la estamos sometidos en nuestra vida diaria y a favor de la calidad de vida. Así lo refleja el Manifesto Slow Food: "contra la locura universal de la 'Fast-Life', se hace necesario defender el tranquilo placer material (...). Comencemos desde la mesa con la 'Slow-Food', contra el achatamiento producido por la 'Fast-Food', y redescubramos la riqueza y los aromas de la cocina local'. 
Con base en los principales conceptos de Slow Food, se fundó posteriormente en Italia la asociación Cittaslow -Rete Internazionale delle città del buon vivere. El objetivo es ampliar la filosofía de Slow Food a las comunidades locales y el gobierno de las ciudades aplicando la búsqueda de la calidad de vida a través de la actitud a favor de lo local, de las tradiciones culturales y de la defensa de la lentitud.

Por tanto, se trata de un movimiento internacional con base en el nivel local que defiende la vida en la città como ideal de vida y propone una serie de criterios para alcanzarlo. De hecho, para formar parte de esta red, las ciudades que quieran incorporarse deben cumplir con una serie de compromisos concretos y verificables -como apoyar la agricultura, gastronomía y el comercio local, mejorar el sistema de acogida de turistas así como recuperar centros históricos entre otros-, comunes para todos los miembros de la red y que funcionan como certificado de calidad, además del requisito de no superar los 50.000 habitantes.

Se puede considerar que Cittaslow no está dirigida en primer término al turismo o marketing de destino sino al patrimonio local, al medioambiente y a la economía social (Nilsson et al., 2007). No obstante, la implicación de la población local, su hospitalidad y la calidad de vida derivada de la asunción de los compromisos del movimiento sirven como herramienta para el desarrollo de un producto turístico. En este sentido, Cittaslow como certificación o etiqueta tiene un efecto potencial de marketing para las localidades que la adquieran (Nilsson et al, 2007).

\section{El turismo Slow}

En un contexto global de consumismo y de cambio climático, existen ciertas tendencias sociales que apuntan hacia un consumidor más consciente que pone en tela de juicio el actual sistema de consumo despilfarrador, alienante y dañino tanto para el medio ambiente como para la propia sociedad. En esta dirección, en el campo de la práctica turística surge el turismo Slow como alternativa de la forma generalizada del consumo vacacional. Esta alternativa abarca a todos los actores de mercado, tanto consumidores como productores, por lo que conlleva implícitamente un cambio global, tanto en el campo económico como cultural. Dicho de otra forma, el turismo Slow requiere las responsabilidades de todos los actores del mercado y pasa por una transformación del imaginario dominante.

Por tanto, el turismo Slow es una forma de considerar tanto el viaje turístico como el tiempo vacacional en el destino como partes integradas de la experiencia vacacional, de acuerdo a una filosofía -Slow- que implica un consumo responsable, la percepción del ocio y el turismo como satisfacción y recuperación tanto física como psíquica, cultural y social y la convicción de que la calidad debe primar sobre la cantidad. Es decir, el turismo Slow va a suponer el uso de tecnología eco-eficiente -rechazo del avión a favor de otros medios de transporte menos contaminantes- por parte de un consumidor responsable, que pretende la reducción de su demanda y la reducción del gasto energético, de materias primas y de la emisión de CO2 -viajes más locales y de mayor duración-, y cuyo objetivo es disfrutar de su 
tiempo de ocio para recuperarse, desarrollarse como persona y aprender durante el encuentro humano en el destino.

\section{a). La oferta del turismo Slow}

Ante la filosofía en que se encuentra parece lógico pensar que la oferta desarrollada en torno al turismo Slow debe animar a que los turistas permanezcan más tiempo en el destino para que el concepto cobre sentido (Matos, 2002). Además, una mayor duración de la estancia podría suponer una mayor interacción con la población local con lo que podría aumentar el valor añadido del viaje además de ser beneficioso para ambas partes implicadas en términos de comprensión recíproca. De acuerdo con Matos (2002), para promover y desarrollar el turismo Slow de manera exitosa se requiere que todos los interesados directa o indirectamente, estén informados, motivados e implicados. Así, es imprescindible que la población local acepte el turismo Slow al igual que es necesaria la implicación de empresas y asociaciones locales El turismo Slow podría mejorar, en la medida de lo posible, los productos turísticos ya existentes en un destino en lugar de crear toda una gama de nuevos productos (Matos, 2002). Se trata de promover el producto local, los métodos tradicionales y artesanales, los bienes naturales e incluso la vivienda rural.

Matos (2002) defiende el turismo Slow como un tiempo de vacaciones para la recuperación y la recreación cercanas al entorno natural donde la lentitud y tranquilidad juegan un papel fundamental para alcanzar el concepto de bienestar. Consecuentemente, bajo su visión del turismo Slow incluye el alojamiento, la dieta, la recreación, la cultura, los servicios y el respeto por el medio ambiente. Puesto que defiende valores cercanos a la naturaleza, el autor ubica en entornos rurales la práctica del turismo Slow. El alojamiento por tanto, se espera que sea rural.

\section{b). La demanda del turismo Slow}

De una forma general y de acuerdo con los resultados de Dickinson et al (2010), en cuanto al viaje turístico Slow parece que persisten ciertos cánones que inducen al turista a asumir automáticamente que volará a determinados destinos. Ésta es una de las principales diferencias entre el turista Slow y el turista no Slow: el turista no Slow concede prioridad a la elección del destino mientras que el turista Slow considera en primer lugar el medio de transporte y posteriormente el destino.

Asimismo, el estudio de Babou y Callot (2007) pone de manifiesto que entre los nuevos segmentos del turismo detectados por los autores, el del turismo Slow es uno de los más prometedores. Las principales características del mismo identificadas por estos autores giran en torno a la valoración del capital tiempo y espacio que permitirán el descubrimiento del destino así como de uno mismo.

\section{Las Cittaslow en España}

En la actualidad, la red Cittaslow se ha extendido por el resto de continentes y está presente en numerosos países. En España las primeras certificaciones de ciudades lentas 
aparecen en 2008. Hoy en día son seis los municipios integrados en Cittaslow - Red Estatal de Municipios por la calidad de vida en España: Begur y Pals en Girona, Mungia y Lekeitio en Vizcaya, Rubielos de Mora en Teruel y Bigastro en Alicante.

En lo referente a la comunicación, todos estos municipios distan como máximo a una hora en coche de otros núcleos de población importantes como capitales de comarca o de provincia. Tan sólo Rubielos de Mora dispone de estación de tren, pero la misma se encuentra a $17 \mathrm{Km}$. del núcleo poblacional principal. En el resto de municipios la máxima distancia es de $31 \mathrm{~km}$.

En cuanto a la población, se trata de pequeños municipios en los que tan sólo en uno de ellos la población supera los 10.000 habitantes, Mungia. El número en general aumenta, salvo en el municipio de Lekeitio. No obstante, su crecimiento vegetativo o natural es negativo -excepto en Mungia y Bigastro- por lo que la inmigración tiene una repercusión positiva en cuanto al crecimiento real de la población de estos lugares así como para la economía local. En municipios como los de Begur, Pals, Bigastro y Rubielos de Mora la población extranjera afincada en los mismos supone más del $10 \%$ del total de la población hasta llegar al $27 \%$ en Pals. Sin embargo, cabe destacar que en algunos de estos casos una buena parte de la población extranjera que se asienta en estos lugares corresponde con personas mayores de 65 años.

En cuanto al nivel de bienestar y calidad de vida se observa en general una buena gestión de políticas públicas. En lo referente a la estructura económica, el sector servicios muestra unos valores muy significativos en términos de generación de empleo y de valor añadido bruto en prácticamente todos los municipios. Es este un reflejo del ritmo de crecimiento de los núcleos poblacionales y del propio turismo. La industria también se presenta como sector relevante en este contexto económico en detrimento de la agricultura.

Finalmente, el turismo parece estar presente con distinta relevancia en estos municipios. Begur y Pals parecen ser dos municipios de carácter turístico -así se ha podido observar a través de sus páginas web, del número de plazas hoteleras y del número de viviendas de segunda residencia. El resto de municipios parecen haberse incorporado recientemente a la oferta turística estatal y así lo demuestra la evolución de plazas de alojamiento en los últimos años y la previsión de nuevos establecimientos de alojamiento. En cuanto a tipología, predomina la oferta hotelera frente a la oferta de vivienda rural. Incluso en uno de los municipios -Begur- no existen infraestructuras de alojamiento rural.

\section{METODOLOGÍA}

Nuestro propósito es arrojar un poco de luz sobre el tema de nuestro trabajo por lo que consideramos acertada la investigación de tipo cualitativa dada su finalidad de "comprensión de una realidad" (Ruiz Olabuénaga, 1996: 13), si bien ello no impide la complementariedad de la misma con métodos cuantitativos a los que hemos considerado oportuno recurrir llegado el momento. Para la obtención de la información empírica se ha optado por las entrevistas telefónicas en profundidad no estructuradas a aquellos sujetos 
que tienen un papel esclarecedor dentro de la gestión de estos municipios en general y del área turística de cada uno de ellos en particular.

\section{RESULTADOS}

De acuerdo con los resultados obtenidos y de forma global, podemos decir que existen diferencias entre los municipios costeros -Begur, Pals y Lekeitio- y los de interior Mungia y Rubielos de Mora-. Bigastro es un municipio de interior pero que se encuentra muy próximo a la costa y está entre las dos principales caracterizaciones que hemos identificado. Los municipios del litoral parecen tener una mayor trayectoria y peso turístico frente a los de interior. Los trazos comunes en los municipios del conjunto costero corresponden, en primer lugar, a la trascendencia del turismo extranjero en el cual las nacionalidades europeas parecen ser las más habituales. Por su parte, en Rubielos de Mora y en Mungia apenas se registra la llegada de turistas extranjeros.

En segundo lugar, en relación con la edad de los turistas tan sólo en el caso de Mungia se ha especificado que mucha gente joven hace turismo en el municipio. En el resto de los casos parece predominar los "matrimonios de mediana edad", las "parejas de mediana edad con hijos" o las "familias con hijos", "abuelos con los nietos", los "matrimonios de la tercera edad" $\mathrm{y}$, a lo sumo, "parejitas" que entendemos como parejas de gente joven.

En tercer lugar, en los municipios del litoral, incluido Bigastro, tiene lugar el veraneo, asociado en gran medida al alojamiento en una casa en propiedad por parte de turistas de origen principalmente extranjero.

En cuarto lugar, en estos municipios costeros, tal y como ocurre en muchos otros del resto de España y en este caso también en Rubielos de Mora, se produce una excesiva concentración de gente en momentos puntuales como verano, semana santa y otras festividades. En el resto de casos no se aprecia ningún efecto negativo resultante de la actividad turística. Es más, en general, el turismo es visto como un fenómeno positivo cuyos efectos van más allá de la perspectiva económica y se transforman en un sentimiento de orgullo del lugar y de la cultura propia.

Finalmente, un hecho compartido por los seis municipios es el medio de transporte mediante el que el turista llega al destino. En todos los casos el coche predomina. Las principales razones son que no existe ninguna estación de tren en el mismo núcleo poblacional y/o que el servicio de autobuses es deficitario. Asimismo, y a consecuencia de esto, el coche también es requerido para los desplazamientos una vez en el destino para conocer o desplazarse a otras localidades.

En cuanto a la motivación principal para incluirse en la red Cittaslow se hace alusión reiteradamente a la filosofía Slow:

"[Nuestro municipio] decidió adherirse al movimiento slow porque comparte la filosofía de que los pueblos empiezan a perder su 
idiosincrasia y los elementos que definen su identidad con la globalización".

En muchos casos el certificado Cittaslow pasa a ser un sello de garantía de calidad tanto para el autóctono como para el visitante al reflejar un estilo y filosofía de vida propios.

En cuanto a la relación entre la filosofía del movimiento Cittaslow y el turismo se nos ha asegurado que sí existe una relación directa pero se ha querido hacer hincapié en que Cittaslow no tiene, al menos de entrada, un fin turístico:

"Sí existe una relación directa. Puede ser y es un reclamo turístico pero es importante matizar que no se trata de una marca turística y que descartamos a los municipios considerados destinos turísticos de masas"

Asimismo en otra entrevista se nos ha hecho ver que el turismo está implícito en la filosofía de Cittaslow. Por otra parte quisimos indagar si el hecho de formar parte de la red Cittaslow supone realmente un reclamo turístico Por un lado, se nos ha dicho que no se persigue que cambie el turismo en el municipio; por otro, dos de las personas entrevistadas no consideran que haya pasado el tiempo suficiente como para hacer esa valoración, mientras que otras personas sí han apreciado cambios en la llegada de turistas a su municipio.

Finalmente, hemos preguntado si conocen el turismo Slow o si lo han escuchado en alguna ocasión, con el objetivo, en primer lugar, de comprobar si es un tema todavía poco conocido y, en segundo lugar, con el objetivo de comprobar si asocian el término a Cittaslow. Tan sólo en dos municipios se nos ha contestado afirmativamente pero el significado parece no estar muy claro.

\section{CONCLUSIONES}

El estudio de los municipios incluidos en la red Cittaslow de España muestra que la realidad turística de los mismos no resulta ajustarse del todo a la práctica de un turismo como es el Slow, de acuerdo con la filosofía y literatura que hemos recogido y comentado sobre el tema en el marco teórico.

En primer lugar, en estos municipios todavía se están llevando a cabo labores muy iniciales en torno al establecimiento de unas bases férreas para los requisitos que otras localidades candidatas deberán cumplir para adherirse a la red. Consecuentemente, se encuentran en una fase temprana de trabajo relativo a todos los grandes bloques temáticos sobre los que trabajan las Cittaslow. Por tanto, cabe esperar para ver cómo cambia su oferta de cara al turismo en el futuro y comprobar si se focalizan en el turismo Slow verdaderamente como una filosofía de actuación o como una mera estrategia de marketing. La demanda es una de las partes clave para que exista turismo Slow. Y en este caso los turistas parecen no ajustarse a su filosofía: una parte considerable de los turistas que visitan estos municipios son extranjeros que para llegar a sus destinos utilizan el avión como medio 
de transporte; empleo del coche para todos los desplazamientos; las estancias de los turistas españoles son, en general, de corta duración, etc. No obstante, se hace necesario un estudio de la demanda que identifique de forma más concreta el perfil del turista Slow para tener una visión más nítida del turismo Slow.

En relación a la estancia, en algunos de los municipios se producen estancias largas, como sería lo deseable de acuerdo con la teoría del turismo Slow, pero tienen que ver en muchos casos con el turismo residencial. Existe, por tanto, el riesgo de que el turismo Slow sea entendido como la práctica de turismo residencial. Así pues, el riesgo potencial es el aumento de la construcción de segundas viviendas en las localidades en cuestión.

A modo de conclusión final, el turismo Slow hace alusión fundamentalmente a una actitud. El turista Slow es un turista predispuesto a la consecución de una experiencia turística de calidad sin menoscabar el peso de sus acciones en términos ambientales y humanos. Es por ello que la oferta turística también debe tener presente la filosofía del turismo Slow y actuar en consecuencia.

No obstante, no hemos detectado por completo que alguna de las partes, oferta y demanda, haya comprendido realmente esta ideología y la haya puesto en práctica. Por tanto, hoy por hoy, las Cittaslow de España no se adecuan perfectamente para la práctica del turismo Slow. Sin embargo, esto no implica que esta práctica turística no pueda tener lugar en otros destinos, sean Cittaslow o no. Es más, dada la naturaleza limitada de esta investigación tampoco sabemos qué ocurre en el resto de redes extranjeras de Cittaslow. Todo ello sin olvidar que el fin último de las Cittaslow no es el turismo sino la calidad de vida de sus habitantes.

Ahora bien, dado el carácter novedoso del turismo Slow y la adhesión reciente de los municipios españoles a la red Cittaslow debemos esperar para ver cómo evolucionan ambas partes.

\section{REFERENCIAS BIBLIOGRÁFICAS}

BABOU, I. \& CALLOT, P. (2007, noviembre). CO2 et tourisme: vers de nouvelles segmentations? Artículo presentado en las $12 \%$ Jornadas de Investigación en Marketin de Bourgogne. Dijon, Francia

CASTILLO, J. (1987). Sociedad de consumo a la española. Madrid: Eudema.

DICKINSON, J.E., ROBBINS, D. \& LUMSDOM, L. (2010). “Holiday travel discourses and climate change". Journal of Transport Geography, 18, 482-489.

HONORÉ, C. (2009). Elogio de la lentitud. Un movimiento de alcance mundial cuestiona el culto a la velocidad. Barcelona: RBA Libros.

LATOUCHE, S. (2006). “iAbajo el desarrollo sostenible! iViva el decrecimiento convivencial!” En Colectivo revista Silence. Objetivo decrecimiento ¿Podemos seguir creciendo hasta el infinito en un planeta finito? Barcelona: Lector.

LATOUCHE, S. (2008). La apuesta por el decrecimiento ¿Cómo salir del imaginario dominante? Barcelona: Icaria. 
MATOS, R. (2002). "Can "slow tourism" bring new life to mountain areas?" Artículo presentado en la conferencia Leisure Futures. Universidad de Innsbruck.

NILSSON, J.H., SVÄRD, A., WIDARSSON, Å. \& WIRELL, T. (2007). "'Slow" Destination Marketing in Small Italian Towns". Artículo presentado en el 16 Nordic Symposium in Tourism and Hospitality Research. Helsingborg, Suiza.

NORBERG-HODGE, H. (2006). "De la dependencia mundial a la interdependencia local". En Colectivo revista Silence. Objetivo decrecimiento ¿Podemos seguir creciendo hasta el infinito en un planeta finito. Barcelona: Leqtor.

RUIZ OLABUÉNAGA, J.I. (1996). Metodología de la investigación cualitativa. Bilbao: Universidad de Deusto.

SCHNEIDER, F, KALLIS, G \& MARTINEZ-ALIER, J. (2010). "Crisis or opportunity? Economic degrowth for social equity and ecological sustainability. Introduction to this special issue", Journal of Cleaner Production, 18, 511-518.

ZORRILLA, R. (1990). El consumo del ocio. Una aproximación a la teoría del tiempo libre desde la perspectiva del consumo. Basauri: Administración de la Comunidad Autónoma del País Vasco. 